\title{
Integrated silicon spectrometers gain resolution, usability
}

Pavel Cheben, André Delâge, Adam Densmore, Siegfried Janz, Boris Lamontagne, Jean Lapointe, Edith Post, Jens Schmid, Phillip Waldron, and Dan-Xia Xu, María Luisa Calvo, and Oscar Martínez Matos

Ultracompact broadband integrated components gather light efficiently and separate wavelengths with high spectral resolution.

Spectrometers, both in optical communications and spectroscopy, spatially divide light of different wavelengths. The integrated waveguide version of a spectrometer, the arrayed waveguide grating ${ }^{1}$ (AWG, see Figures 1 and 2), is the key optical device used for this purpose in wavelength-divisionmultiplexed (WDM) optical networks. If arrayed waveguide technology can be adapted for applications in high-resolution spectroscopy, it could free users from relying on large and expensive tabletop spectroscopic instruments. A compact highresolution spectrometer chip similar to the one we developed could be a significant breakthrough.

Silicon is the dominant platform for the microelectronics industry. An important advantage of silicon for making photonic circuits is its large refractive index $(n \sim 3.5)$, which allows for light confinement in waveguides with cross-sections up to 600 times smaller than conventional low-index-contrast glass waveguides. As a result, silicon can be used to make ultracompact planar waveguide circuits. Two of the biggest hurdles facing the introduction of silicon photonic circuits are large losses from both fiber-to-chip coupling and waveguide propagation losses, and sensitivity to polarization.

By taking advantage of the high refractive index of silicon waveguides, we demonstrated a compact silicon AWG with a wavelength resolution of $0.08 \mathrm{~nm}$ and a total chip size of $8 \times 8 \mathrm{~mm}^{2}$ fabricated on a silicon-on-insulator (SOI) waveguide platform (see Figure 1). ${ }^{2}$ A glass waveguide-based device with a comparable resolution developed by NTT occupies a 4in. wafer, two orders of magnitude larger than our spectrometer. We maximized the AWG resolution by reducing the input and output waveguide apertures, as shown in Figure 1(b). This is analogous to narrowing the input and output slit widths in a con-
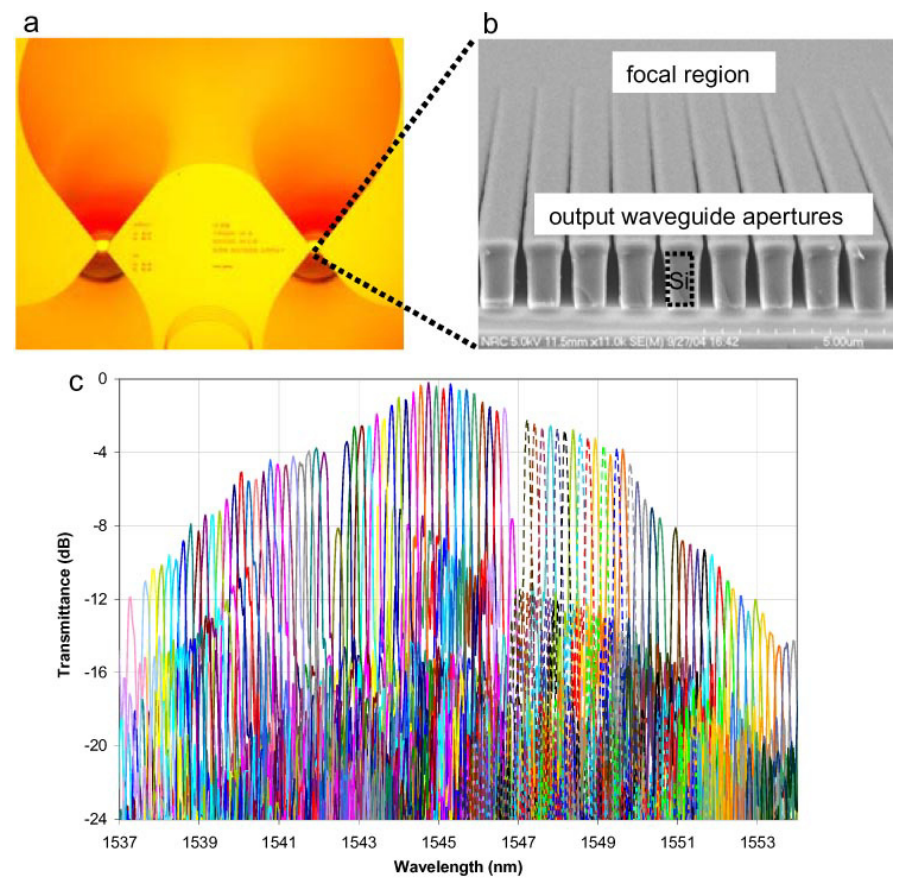

Figure 1. (a) A microphotograph shows a high-resolution silicon arrayed waveguide grating ( $A W G$ ) spectrometer. (b) The device has narrow apertures at the spectrometer's focal region. (c) The device's spectrum shows channels in the mid-IR.

ventional grating monochromator. The measured spectrometer channel spectra are shown in Figure 1(c). We also proposed that the resolution can be increased significantly by inserting a triangular photonic bandgap waveguide section into the waveguide array. Within this section, light propagation is slowed down (see Figure 2). ${ }^{3}$

We also proposed and designed the first Fourier-transform (FT) Michelson-type AWG spectrometer. ${ }^{4}$ Its main benefit is a markedly increased light throughput, or étendue. Unlike conventional FT spectrometers, our device does not need scanning mirrors. The spectrometer is formed by two interleaved AWGs that

Continued on next page 


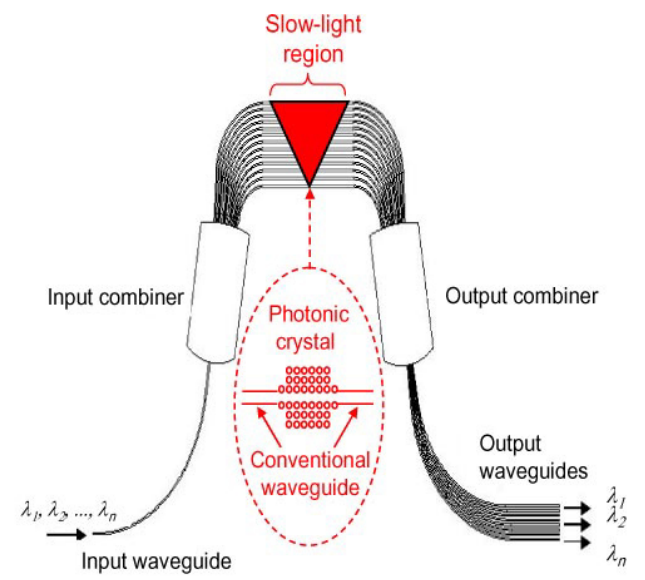

Figure 2. An AWG chip includes a triangular region with slowlight photonic-crystal waveguides, which increase the device's spectral resolution.

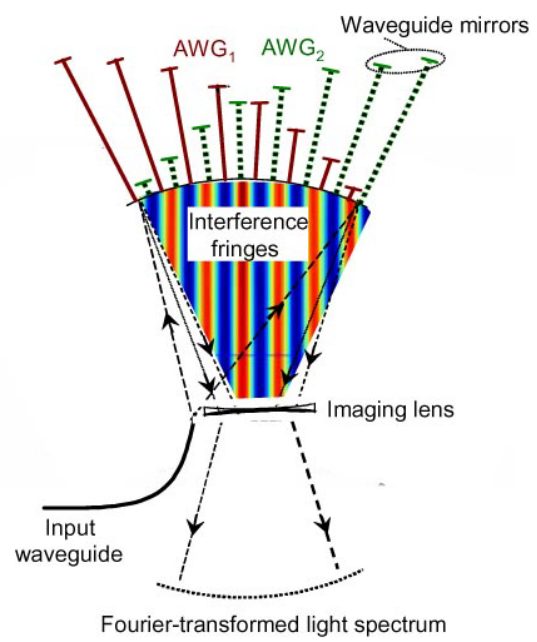

Figure 3. Two interleaved arrayed waveguide gratings $\left(A W G_{1}\right.$ and $A W G_{2}$ ) create interference fringes in a Fourier-transform (FT) AWG spectrometer, which offers large light throughput.

produce interference fringes with different spacing for different wavelengths (see Figure 3). The light spectrum is obtained by Fourier analysis of the interference fringes. Our instrument had a measured spectral resolution of $0.07 \mathrm{~nm}$. This is equivalent to a conventional FT spectrometer with a mirror scanning range of $\sim 10 \mathrm{~mm}$, which is beyond the capability of current MEMS (microelectromechanical systems) technology.

Due to the large mode-size disparities, the coupling of light from the external world (via an optical fiber) to a silicon waveguide chip is inefficient. We proposed two solutions. The first is a planar waveguide graded-index (GRIN) fiber-to-chip coupler, ${ }^{5}$ which acts as the planar analog of a conventional cylindrical (a)
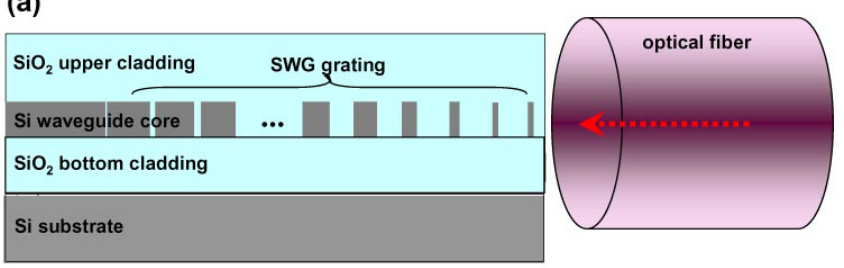

(b)

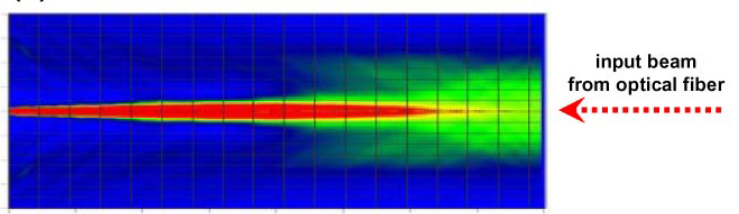

Figure 4. (a) A nonuniform subwavelength grating incorporated into the waveguiding layer in a fiber-to-chip coupler increases efficiency by matching the fiber mode size at the fiber end and the waveguide mode size at the waveguide end of the device. (b) A finite difference time domain simulation of the device with this grating shows efficient coupling.

GRIN lens. Our second coupling approach relies on a subwavelength grating (SWG). ${ }^{6}$ The waveguide's effective index is altered by chirping the SWG duty ratio, as shown in Figure 4(a), so that the fraction of volume filled by the waveguide core material gradually changes. At one end of the coupler, the mode size is matched to the silicon waveguide, while near the chip facet it matches that of the optical fiber. Unlike diffraction-based waveguide grating couplers, the SWG coupler is nonresonant and hence insensitive to both wavelength and polarization. The calculated coupling efficiency-see Figure 4(b)—from an SMF28 fiber to a $0.3 \mu \mathrm{m}$-thick SOI waveguide is $76 \%$. We also found that the coupler is robust in cases of fiber misalignment. ${ }^{6}$

Polarization sensitivity is another critical issue in AWG applications. ${ }^{1}$ We demonstrated that stress-induced birefringence of an $\mathrm{SiO}_{2}$ cladding layer can eliminate the birefringence of a silicon waveguide. ${ }^{7}$ Birefringence-free operation was demonstrated by controlling the $\mathrm{SiO}_{2}$ cladding thickness, or by modifying stress in the oxide film by controlling deposition conditions or by thermal annealing. We used the technique to eliminate polarization-dependent wavelength shift in a nine-channel $200 \mathrm{GHz}$ AWG demultiplexer ${ }^{7}$ and ring resonators.

Silicon AWG technology has made significant progress toward practical multiplexer and spectrometer devices. We have developed compact silicon AWG spectrometers as well as techniques that allow us to markedly increase spectral resolution, light throughput, and the efficiency of light coupling into the

Continued on next page 
chip. We have also found a way to eliminate polarization sensitivity in these devices. Our future research will focus on further advancing silicon AWG technology and on its use in WDM networks, optical interconnects, and biological and chemical sensing. For example, we are now developing a lab-on-a-chip spectrometer integrated with biosensor arrays and readout circuits, as well as a large-throughput FT AWG spectrometer for spaceborne environmental monitoring.

This work has been supported in part by the National Research Council of Canada Genome and Health Initiative.

\section{Author Information}

\section{Pavel Cheben, André Delâge, Adam Densmore,}

Siegfried Janz, Boris Lamontagne, Jean Lapointe, Edith Post, Jens Schmid, Phillip Waldron and Dan-Xia Xu

Institute for Microstructural Sciences

National Research Council of Canada

Ottawa, Canada

Pavel Cheben is a research officer at the Institute for Microstructural Sciences at the National Research Council of Canada, as well as an honorary professor at the Complutense University of Madrid. His research interests include integrated optics, silicon photonics, and optical materials. He was a member of the team that started Optenia and developed the first commercial waveguide echelle-grating WDM demultiplexer. Before joining the NRC, he led the foundation of the Laboratory of Space Instrumentation at the National Institute for Aerospace Technology in Spain.

\section{María Luisa Calvo and Oscar Martínez Matos}

Departamento de Óptica Facultad de Ciencias Físicas

Universidad Complutense

Madrid, Spain

\section{References}

1. P. Cheben, Wavelength dispersive planar waveguide devices: echelle gratings and arrayed waveguide gratings, in M. L. Calvo and V. Lakshminarayanan (eds.), Optical Waveguides: From Theory to Applied Technologies, pp. 173-232, Taylor and Francis, London, 2006.

2. P. Cheben, A. Bogdanov, A. Delâge, S. Janz, B. Lamontagne, M. J. Picard, E. Post, and D.-X. Xu, A 100-channel near-infrared SOI waveguide microspectrometer: design and fabrication challenges, Proc. SPIE 5644, pp. 103-110, 2005.

3. O. Martínez, M. L. Calvo, P. Cheben, S. Janz, J. A. Rodrigo, D.-X. Xu, and A. Delâge, Arrayed waveguide grating based on group index modification, J. Lightwave Technol. 24, pp. 1551-1557,2006.

4. P. Cheben, I. Powell, S. Janz, and D.-X. Xu, Wavelength dispersive device based on a Fourier-transform Michelson-type arrayed waveguide grating, Opt. Lett. 30, pp. 18241826, 2005.

5. A. Delâge, S. Janz, B. Lamontagne, A. Bogdanov, D. Dalacu, D.-X. Xu, and K. P. Yap, Monolithically integrated asymmetric graded and step-index couplers for microphotonic waveguides, Opt. Express 14, pp. 148-161, 2006.

6. P. Cheben, D.-X. Xu, S. Janz, and A. Densmore, Subwavelength waveguide grating for mode conversion and light coupling in integrated optics, Opt. Express 14, pp. 46954702, 2006.

7. D.-X. Xu, P. Cheben, D. Dalacu, A. Delâge, S. Janz, B. Lamontagne, M. J. Picard, and W. N. Ye, Eliminating the birefringence in silicon-on-insulator ridge waveguides by use of cladding stress, Opt. Lett. 29, pp. 2384-2386, 2004. 